\title{
Optical observations of Very High Energy Sources from the Boyden Observatory
}

\section{B. van Soelen*}

University of the Free State, Nelson Mandela Drive, Bloemfontein, 9301, South Africa E-mail: wansoelenoduts.ac.za

\section{I.P. van der Westhuizen}

University of the Free State, Nelson Mandela Drive, Bloemfontein, 9301, South Africa E-mail: VanDerwesthuizenIPdufs.ac.za

\section{P.J. Meintjes}

University of the Free State, Nelson Mandela Drive, Bloemfontein, 9301, South Africa E-mail: meintjpjeufs.ac.za

\section{Hanlon}

School of Physics, University College Dublin, Belfield, Dublin 4, Ireland

E-mail: Lorraine.hanlonducd.ie

\section{Murphy}

School of Physics, University College Dublin, Belfield, Dublin 4, Ireland E-mail: david.murphy.5eucdconnect.ie

\section{A. Martin-Carrillo}

School of Physics, University College Dublin, Belfield, Dublin 4, Ireland

E-mail: antonio.martin-carrilloducd.ie

\section{Klindt}

University of the Free State, Nelson Mandela Drive, Bloemfontein, 9301, South Africa E-mail: lizelkeklindtegmail.com

\section{H.J. van Heerden}

University of the Free State, Nelson Mandela Drive, Bloemfontein, 9301, South Africa E-mail: VanHeerdenHJdufs.ac.za 
Multi-wavelength observations of very high energy sources can place better constraints on the emission processes at work in these systems as well as the location of this emission. For example, the spectral energy distributions of Active Galactic Nuclei (AGN) display two distinct components that radiate at lower (radio to UV/X-ray) and higher (X-ray to gamma-ray) energies. If the emission is the result of a simple one zone model, correlations should be observable between these different components. Such correlations are, however, more complicated than a simple linear relation, but show variations based on colour and spectral distribution. It is therefore important to undertake multi-wavelength observations of known $\mathrm{TeV}$ gamma-ray sources to establish multiwavelength correlations. We are beginning a long term optical monitoring campaign of known TeV AGN/Blazars, from the Boyden Observatory, using, in particular, the Watcher Robotic Telescopes. Additional observations are capable with the Boyden 1.5-m telescope as well as with the 20-inch telescope recently obtained from the South African Astronomical Observatory. We present an overview of the new monitoring campaign which is being undertaken as well as a brief overview of the Boyden Observatory, and the status of the available telescopes.

3rd Annual Conference on High Energy Astrophysics in Southern Africa-HEASA2015, 18-20 June 2015

University of Johannesburg, Auckland Park, South Africa

*Speaker. 


\section{Introduction}

Multi-wavelength astronomy places important constraints on the underlying physics of astronomical sources. Lower energy (radio to optical) multi-wavelength observations provide significant information which can complement the high energy observations (X-ray to gamma-ray) of sources observed by satellites (e.g. Fermi and AGILE) and ground based Imaging Air Cherenkov Telescopes (e.g. H.E.S.S., MAGIC, VERITAS). South African observatories are well placed to provide such multi-wavelength (optical and radio) observations, through the current facilities (e.g. South African Astronomical Observatory, Boyden Observatory, Hartebeesthoek Radio Astronomy Observatory, KAT-7) as well as future developments (e.g. Square Kilometre Array). This is particularly true for the H.E.S.S. telescope, since it is located in Namibia, making simultaneous radio, optical and VHE observations of sources possible. The requirement for lower energy, multi-wavelength observation of Very High Energy (VHE, $>100 \mathrm{GeV}$ ) sources will increase with the development of the Cherenkov Telescope Array (CTA), which will greatly increase the observational sensitivity at VHE.

There are currently ${ }^{1} 172$ known TeV sources listed in the TeVCat. ${ }^{2}$ These observations include detections of Pulsar Wind Nebula, pulsars, binary systems and Active Galactic Nuclei (AGN). Approximately 40 per cent of sources are associated with AGN (compared to 75 per cent that are associated with AGN in the recently released Second Catalog of Hard Fermi-LAT sources (2FHL) [四). However, gamma-ray bursts (GRBs), which are regularly detected at $\mathrm{GeV}$ energies, have yet to be detected at $\mathrm{TeV}$ energies.

Here, we briefly review the available facilities available at the Boyden Observatory (Section \) and report on a recent monitoring campaign begun to provide additional optical constraints on known TeV AGN sources, using the Watcher Robotic Telescope [ᄆ] (Section [1]).

\section{The Boyden Observatory}

The Boyden Observatory was originally established as a southern station of the Harvard College Observatory, in Peru, through funding provided by the Boyden Fund in 1887 [ [3]. The observatory moved to its current location, approximately $25 \mathrm{~km}$ North-East of Bloemfontein, South Africa ( $\left.29^{\circ} 02^{\prime} \mathrm{S}, 26^{\circ} 24^{\prime} \mathrm{E}\right)$ between 1927 to 1933 [䧃].

The Boyden Observatory, operated by the Department of Physics at the University of the Free State (UFS), currently hosts two science telescopes, the UFS-Boyden 1.5-m telescope and the Watcher Robotic Telescopes (which is operated by the University College of Dublin). A third, the 0.5-m Boller \& Chivens telescope, which was decommissioned at the SAAO in January 2015, is currently being installed, and is close to being operational.

\subsection{UFS-Boyden 1.5-m telescope}

UFS-Boyden $1.5-\mathrm{m}$ reflector is in a Cassegrain configuration, with a $1.5 \mathrm{~m}$ diameter primary mirror and a $23.82 \mathrm{~m}$ focal length (f/15.6). Photometric observations are undertaken using an Apogee U55 Back-illuminated CCD camera $(770 \times 1152$ pixel array), which records a field of

\footnotetext{
${ }^{1}$ On 15 October 2015.

${ }^{2}$ http://tevcat.uchicago.edu/
} 
view of $2.5 \times 3.7$ arcmin. The filter wheel is equipped with $\mathrm{U}, \mathrm{B}, \mathrm{V}, \mathrm{R}_{\mathrm{c}}, \mathrm{I}_{\mathrm{c}}$ and Clear filters. The telescope is driven via a DFM Engineering control system ${ }^{3}$ and interfaced through the custom written SPICA program.

\subsection{Watcher Robotic Telescope}

The Watcher Robotic Telescope, is a dedicated Gamma-ray Burst (GRB) monitoring telescope, and forms part of the GLORIA project [5] ${ }^{4}$ The telescope system is connected to the Gammaray Coordination Network (GCN) and can automatically slew and begin observations of potential GRB sources within $\sim 60$ seconds. The telescope system was established by UCD (Ireland) in partnership with AsÚ AVČR (Czech Republic), IAA (Spain) and the UFS. The system is currently equipped with an Andor iXon 888 electron-multiplying CCD $(1 \mathrm{k} \times 1 \mathrm{k})$ with clear, V, R, $\mathrm{g}^{\prime}, \mathrm{r}^{\prime}, \mathrm{i}^{\prime}$, $\mathrm{H}-\alpha$, and OIII filters, and a $10 \times 10$ arcmin field of view. ${ }^{5}$ The telescope system is shown in Fig. $\mathrm{W}$.

Recent observations with Watcher of GRB 120711A were reported by [G]. Time not used for GRB observations is used to observe, in particular, blazar sources. Observations have also recently been reported on the cataclysmic variable ASASSN-15ni [ [U] and the low-mass X-ray binary V404 Cyg [ [8], Q Q].

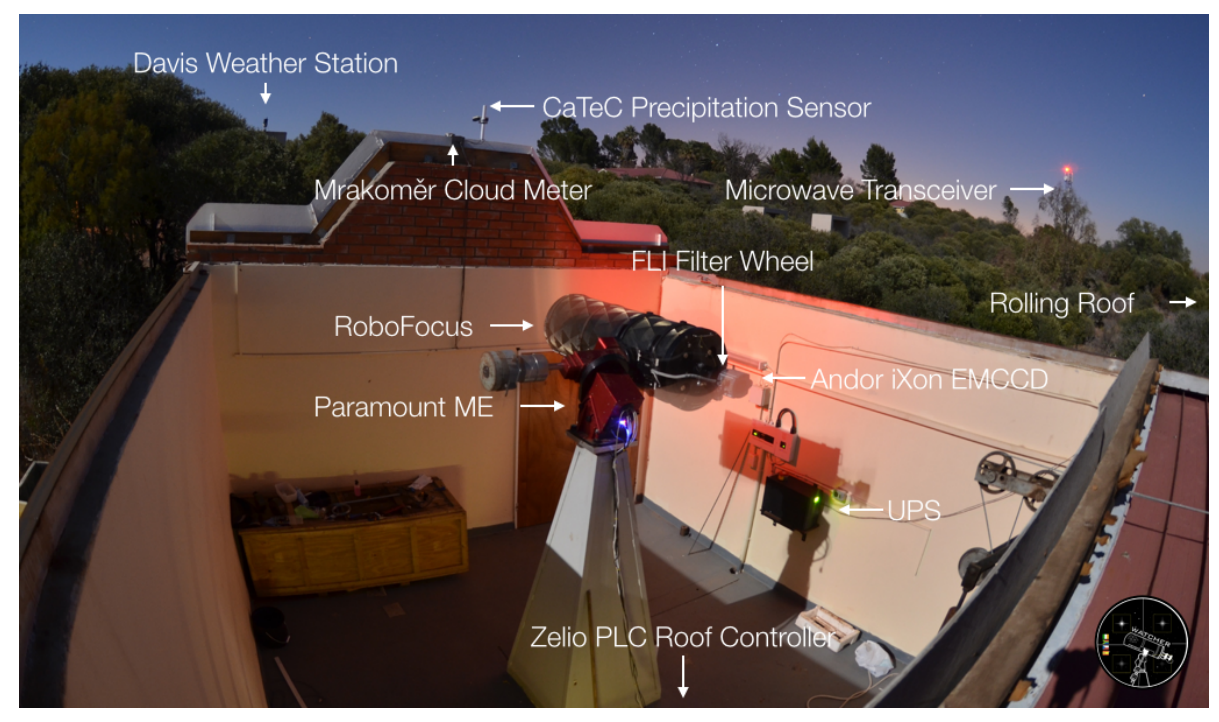

Figure 1: The Watcher Robotic Telescope located at the Boyden observatory. Image credit: D. Murphy.

\subsection{0 -inch telescope}

The most recently installed telescope at the Boyden observatory is the 0.5 -m Boller \& Chivens telescope. It was originally installed at the Republic Observatory in Johannesburg, in 1968, before being moved in 1972 to the Sutherland site of the South African Astronomical Observatory. In 2014/2015 the telescope was decommissioned, was transported to the Boyden observatory in

\footnotetext{
${ }^{3}$ http://www.dfmengineering.com/

${ }^{4} \mathrm{http}: / /$ gloria-project.eu

${ }^{5}$ Further information on the Watcher Telescope is available at http://watchertelescope.ie/.
} 
February 2015, and the base installed on the 12th of May 2015. The telescope is currently in commissioning. The telescope is a Cassegrain (f/18) design and when completed, the system will be equipped with a photoelectric photometer, with $\mathrm{U}, \mathrm{B}, \mathrm{V}, \mathrm{R}_{\mathrm{c}}$ and $\mathrm{I}_{\mathrm{c}}$ filters.

\section{TeV monitoring campaign}

Various studies have looked at optical variability and the correlation to multi-wavelength results (see e.g. [प], [1, [2] for recent examples). In order to complement such observations, we began a long term, multi-colour monitoring campaign using the Watcher Robotic Telescope to monitor known $\mathrm{TeV}$ sources in April 2015. The outline of the monitoring programme and initial results are presented below.

\subsection{Source Selection}

In order to maximize the potential results of the observations an initial set of 17 brighter $(\mathrm{V}=$ 12.81 - 17.75) known TeV emitting AGN were selected for inclusion in the monitoring campaign. All sources are south of Declination $+12^{\circ}$. The sources are included in the Watcher scheduler and set to observe approximately once a day, depending on observational constraints (e.g. weather, GRB alerts). Each visit typically consists of 6 observations, of 1 minute each in the V, R and $i^{\prime}$ bands.

\subsection{Data reduction and analysis}

The standard IRAF procedures were followed to perform bias and flat-field correction (dark current effects are negligible). The standard photometric measurements were done using the IRAF/DAOPHOT packages and aperture optimization was performed using the PHOTCAL/MKAPFILE packages.

Photometry was performed for the target and for comparison stars suggested in the Landessternwarte Heidelberg-Königstuhl AGN finding chart service. ${ }^{6}$ Differential photometry was undertaken following a weighted mean approach [[1]3] where each comparison has been weighted with the mean error $\left(\sigma_{c, i}\right)$ associated with the comparison star over all $N$ frames,

$$
\omega_{c}=\frac{1}{N} \sum_{i=1}^{N} \frac{1}{\sigma_{c, i}^{2}}
$$

The corrected magnitude is calculated by

$$
m_{\text {target,corr }}=m_{\text {target }}-\left(\left\langle m_{c}\right\rangle-M\right)
$$

where $\left\langle m_{c}\right\rangle$ is the weighted mean magnitude of all comparisons stars on a frame, and $M$ is the weighted mean magnitude of all comparison stars over all frames. The error on each corrected data point is calculated as

$$
\sqrt{\sigma_{t}^{2}+\left[\sum_{i=1}^{N}\left(\frac{1}{\sigma_{c, i}^{2}}\right)\right]^{-1}},
$$

where $\sigma_{t}$ is the measured error of the target. When the apparent magnitudes of the comparison stars are known, the mean offset between the instrumental and apparent magnitudes is also corrected for.

\footnotetext{
${ }^{6}$ http://www.lsw.uni-heidelberg.de/projects/extragalactic/charts/
} 


\subsection{Preliminary results}

The preliminary results for the monitoring of AP Lib, PKS 2155-304 and PKS 1510-089 during the second two weeks of May 2015 are shown in Figs. $\square$ to H, respectively. In each figure, the solid blue circles indicate the target, while the open grey boxes show one comparison star. All three sources show indications of short time scale variability as expected for blazar sources. This is particular true for PKS 1510-089 which shows a clear increase of $\Delta V \sim 0.7$ mag in a period of $\sim 10$ days (MJD $\sim 57154.8-57164.7$ ) peaking around MJD 57164. This event is coincident with the increase in TeV gamma-rays reported by the MAGIC gamma-ray telescope on the 18th and 19th of May 2015 [144].

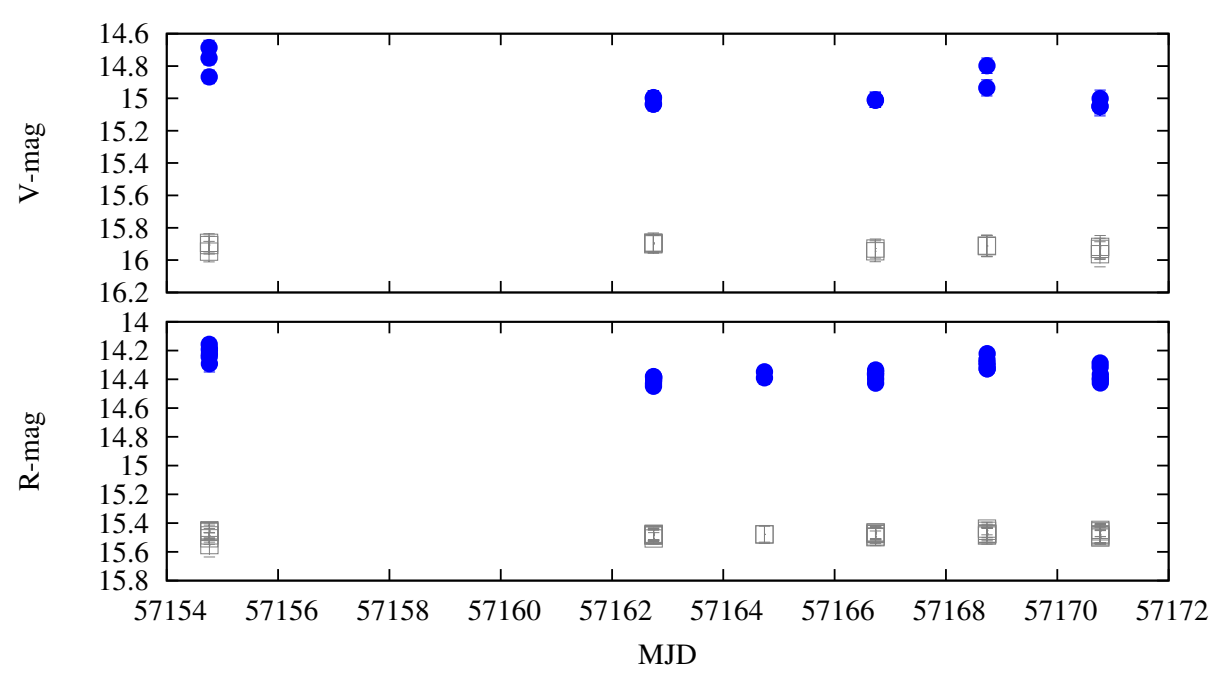

Figure 2: Differential light curve for AP Lib for the second two week in May 2015. The target is indicated by the solid blue circles, while a comparison star is indicated by the open grey boxes.

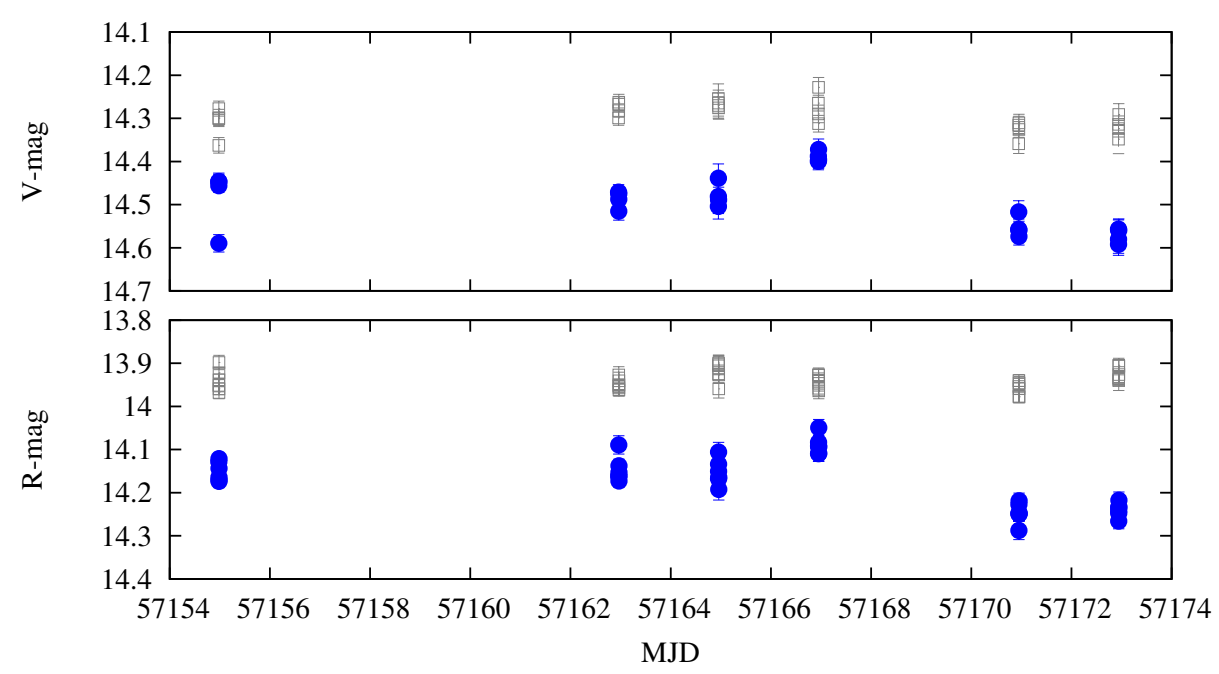

Figure 3: Differential light curve for PKS 2155-304 for the second two week in May 2015. The target is indicated by the solid blue circles, while a comparison star is indicated by the open grey boxes. 


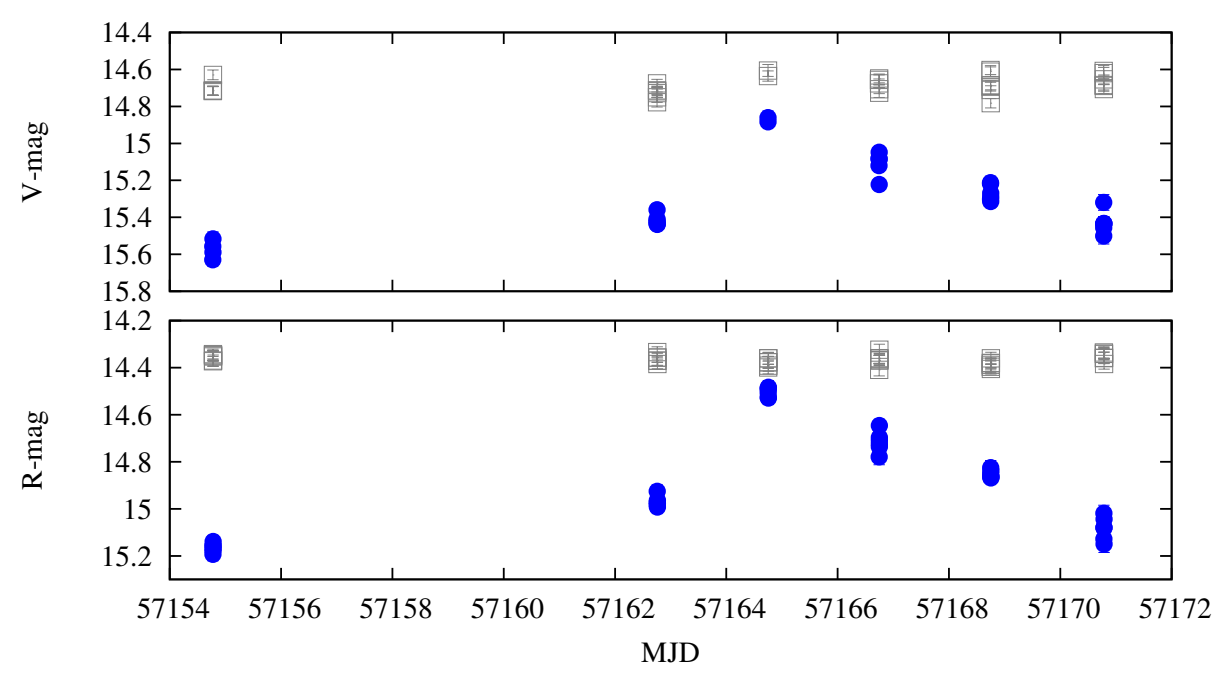

Figure 4: Differential light curve for PKS 1510-089 for the second two week in May 2015. The target is indicated by the solid blue circles, while a comparison star is indicated by the open grey boxes.

\section{Discussion}

The preliminary results shown above present the feasibility of using the Watcher Robotic Telescope for a long term monitoring campaign of AGN targets. The variability detected here is expected as we have purposefully chosen brighter, known variable sources. Previous observations have, for example, found variability of $\Delta R \lesssim 1$ for AP Lib using the ATOM telescope [15]]. For PKS 2155-304, long term optical observations by, for example, [12] have found $\Delta V=2.32$ and $\Delta R=2.41$ over a $\sim 7$ year period and similarly $\Delta R \sim 2$ was found by [[ए5]. Multi-wavelength studies have also recently been reported by, for example, [ए], ㅁ] ]. Similarly, PKS 1510-089, which is one of only a few FSRQs detected at VHE, has had reported optical variability of $\Delta V=2.64$ and $\Delta R=3.43$ over a $\sim 6$ year period [12].

Statistical studies of AGN require large and long term monitoring of sources. The data collected with the Watcher Telescope will provide an additional resource that will contribute towards this aim. It is our intention to extend this monitoring programme beyond this initial phase, by increasing the number of monitored sources, improving the data collection strategies (e.g. better matching of integration times to target brightness), the automation of the data reduction which can provide notification of, for example, flare like events, and providing public access to data.

\section{Conclusions}

Multi-wavelength observations are important to constrain the emission processes in VHE sources. Here we have presented the facilities available at the Boyden Observatory and have briefly presented preliminary results from our planned long term monitoring programme of known very high energy emitting AGN. Initial results show the variability expected for these sources. For one source, PKS 1510-089, an optical flare event has been observed which corresponds to a recently reported increase in $\mathrm{TeV}$ gamma-rays reported by MAGIC [14]]. Observations of these sources are on-going. 


\section{Acknowledgments}

BvS, IPvdW and LK acknowledge the financial assistance of the National Research Foundation (NRF). This work is based on the research supported in part by the National Research Foundation of South Africa for the grant 87919. LH acknowledges support from Science Foundation Ireland (grant 07/RFP/PHYF295) and the EU FP7 (grant agreement no. 283783 ).

\section{References}

[1] M. Ackermann et al. (Fermi-LAT Collaboration), 2FHL: The Second Catalog of Hard Fermi-LAT sources, (2015) arXiv:1503.02664

[2] M Topinka et al., Status update of the Watcher Robotic Telescope, EAS Publications Series 61 (2013) 487

[3] B.Z. Jones \& L.G. Boyd, The Harvard College Observatory, Belknap Press, Cambridge, Massachusetts (1971)

[4] A.H. Jarrett, Boyden Observatory, Occasional Publication, no. 2 of the Institute and Department of Astronomy, University of the Orange Free State (1985)

[5] P. Tisdall et al. Blazar Monitoring with the Watcher Robotic Telescope, in the proceedings of Third Workshop on Robotic Autonomous Observatories, Revista Mexicana de Astronomia y Astrofisica 45 (2014) 71

[6] A. Martin-Carrillo et al. GRB 120711A: an intense INTEGRAL burst with long-lasting soft $\gamma$-ray emission and a powerful optical flash, A\&A 567 (2014) 84

[7] A. Martin-Carrillo et al. Significant colour change of ASASSN-15ni observed with the UCD Watcher telescope, ATel, 7875 (2015)

[8] A. Martin-Carrillo et al. Optical monitoring campaign of V404 Cyg with the Watcher Robotic Telescope, ATel, 7718 (2015)

[9] A. Martin-Carrillo et al. Significant decrease in the average optical emission from V404 Cyg, ATel, 7729 (2015)

[10] K.E. Williamson et al. Comprehensive Monitoring of Gamma-Ray Bright Blazars. I. Statistical Study of Optical, X-Ray, and Gamma-Ray Spectral Slopes, ApJ 789 (2014) 135

[11] A. Abramowski et al. Long-term monitoring of PKS 2155-304 with ATOM and H.E.S.S.: investigation of optical/ $\gamma$-ray correlations in different spectral states, A\&A 571 (2014) A39

[12] A. Sandrinelli, S. Covino, A. Treves Long and short term variability of seven blazars in six near-infrared/optical bands, A\&A 562 (2014) A79

[13] M.E. Everett \& S.B. Howell, A technique for ultrahigh-precision CCD photometry, PASP 113 (2001) 1428

[14] R. Mirzoyan et al. (MAGIC Collaboration), MAGIC detects an increased activity from PKS 1510-089 at very high energy gamma-rays, ATel, 7542 (2015)

[15] A. Wierzcholska et al. Longterm optical monitoring of bright BL Lacertae objects with ATOM: Spectral variability and multiwavelength correlations, A\&A 573 (2015) A69

[16] J. Chevalier et al. Long term variability of the blazar PKS 2155-304, in the proceedings of the ICRC (2015) arXiv:1509.03104 